\title{
Spatial learning by rats across visually disconnected environments
}

\author{
JÉRÔME ROSSIER, MARIE-CLAUDE GROBÉTY, and FRANÇOISE SCHENK \\ Université de Lausanne, Lausanne, Switzerland
}

\begin{abstract}
Experiments were designed in which some properties of spatial representations in rats could be examined. Adult subjects were trained to escape through a hole at a fixed position in a large circular arena (see Schenk, 1989). The experiments were conducted in the dark, with a limited number of controlled visual light cues, in order to assess the minimal cue requirement for place learning. Three light cues identical in shape, height, and distance from the table were used. Depending on the condition, they were either permanently on or alternatively on or off, contingent on the position of the rat in the field. Two questions were asked: (1) How many identical visual cues were necessary for spatial discrimination in the dark, and (2) could rats integrate the relative positions of separate cues, under conditions in which the rat was never allowed to perceive all three cues simultaneously. The results suggest that rats are able to achieve a place discrimination task even if the three cues necessary for efficient orientation can never be seen simultaneously. A dissociation between the discrimination of the spatial position of the goal and the capacity to reach it by a direct path suggests that, with a reduced number of cues, prolonged locomotion might be required for accurate orientation in the environment.
\end{abstract}

Although nocturnal, rats are known to rely mainly on sight to perform most spatial tasks (Fenton, Arolfo, Nerad, \& Bures, 1994; Schenk, Grobéty, \& Gafner, 1997; Zoladek \& Roberts, 1978). A computation based on the integration of several distant visual cues is presumed to allow location of a submerged platform in a circular pool of opaque water (Morris, 1981). Accordingly, place navigation performance is poor in complete darkness (Arolfo, Nerad, Schenk, \& Bures, 1994; Schenk et al., 1997; Sutherland \& Dyck, 1984). Visual cues do not need to be permanently available to support place learning and may be seen only during part of the escape trajectory in the Morris task (Chapillon, 1999; Sutherland, Chew, Baker, \& Linggard, 1987). However, the question remains open as to whether the cues included in a minimal configuration (i.e., a configuration with no redundant cue) must be perceived simultaneously to allow place discrimination or whether they can be integrated over a temporal gap.

Learning a single place might be based on a memory of specific local views, or snapshot memory (Cartwright \& Collett, 1982; Wilkie \& Palfrey, 1987). The possibility that rats rely on the specific panorama of visual cues perceived from the goal area to find the invisible platform suggests that they would perform accurately only by adjusting their ongoing position according to a memorized image of this unique panorama. In agreement with this hypothesis, rats previously trained in a Morris water maze

This research was supported by Grant $3100-039754.93 / 1$ from the Fonds National Suisse de la Recherche Scientifique. Correspondence concerning this article should be addressed to J. Rossier, Institut de Physiologie, Rue du Bugnon 7, 1005 Lausanne, Switzerland (e-mail: jerome.rossier@iphysiol.unil.ch). to escape to a fixed position on the basis of distant visual cues were severely impaired in reaching the same position when the room lights were switched off each time they reached the central region of the pool, where the platform was located (Arolfo et al., 1994). When the size of this central region was reduced, the rats were able to perform the task (Save, 1997), suggesting that their performance depended on the size of the regions from which they perceived the visual environment. A correct discrimination of the escape area was observed in a similar experimental design on a homing table, where rats had to enter a hole at a fixed position relative to distant room cues. Since the room lights were switched off each time they entered the inner region of the table, where the escape hole was situated, the rats did not need to rely on a unique configuration of cues to recognize the training position (Schenk, Grobéty, Lavenex, \& Lipp, 1995). The rats were also capable of discriminating a spatial position in a visually homogeneous arena, which they entered from another region rich in distant visual landmarks (Grobéty, Morand, \& Schenk, in press; Schenk et al., 1997). Thus, rats can memorize a specific position according to visual cues that are no longer available when they reach the target area.

The minimal visual cue requirement has been studied by Fenton et al. (1994) with a limited number of controlled remote visual landmarks. The learning of a new escape position in the Morris navigation task was similar whether four visible cues or only two cues were available in the dark. In fact, latencies were higher in the first training trials, when four cues were available, than in the two-cue condition. This suggests that learning an escape position with a set of four cues requires more behavioral adjustments than when only two cues are present. Adding 
two new cues to a set of two familiar cues significantly delayed escape, whereas removing two cues from a set of four cues had no detectable effect. Thus, new cues seem to induce either a phase of reexploration of the pool or the learning of the relations between the platform location and the location of each new cue. Finally, optimal performance, reached during training with two cues, was abolished when one of these cues was replaced by a new one, suggesting that both cues contributed to the spatial adjustment.

The experiments by Fenton et al. (1994) demonstrate that escape performance is related to the position of controlled visual cues. However, this study was conducted with rats previously trained in a normal illumination condition, which precludes any conclusion as to the value of these sets of cues in the acquisition of the task by untrained rats. Moreover, since the available cues remained permanently on during the task, it is possible that, in these particular conditions, the cues were used to develop accurate visual adjustments to locate the invisible platform. The reaction described above when two new cues were added suggests, indeed, that rats are very dependent on the arrangement of the visual panorama seen from the surface of the water.

The behavioral data are supported by electrophysiological data indicating that, in rats, the firing of hippocampal units is mainly affected by visual inputs. When the light is turned off, the place fields of hippocampal place cells are less stable (Markus, Barnes, McNaughton, Gladden, \& Skaggs, 1994). The spatial firing of hippocampal place units or of postsubicular directional units is most often driven by visual cues during reorientation (Goodridge \& Taube, 1995; Hetherington \& Shapiro, 1997; Knierim, Kudrimoti, \& McNaughton, 1995; Muller \& Kubie, 1987; Taube, Muller, \& Ranck, 1990), but a selection among ambiguous visual cues is based on path integration in relation to a starting reference position (Sharp, Kubie, \& Muller, 1990). Relations among different places are obviously processed thanks to a simultaneous integration of local views and cues derived from the animal's movement (proprioceptive or idiothelic cues; McNaughton, Knierim, \& Wilson, 1995).

Cheng (1986) has also shown that the geometrical structure of the environment or the set of cues is taken into account by rats solving a spatial task (O'Keefe \& Burgess, 1996). The reliability of such a landmark geometry depends on its stability (Biegler \& Morris, 1993, 1996; Greene \& Cook, 1997). This is qualitatively different from the capacities provided by a snapshot memory that would simply help to guide accurate approaches. Therefore, both the behavioral performance and the firing of hippocampal or subicular units suggest that visual cues are integrated into a multimodal spatial representation of their relative position that facilitates the selection of relevant information (Buresova \& Bures, 1981).

The important issue is how different cues contribute to the elaboration of a spatial representation to support accurate place learning. Three cues are necessary to unam- biguously define a place in a nonlimited euclidean space. This is not necessarily the case in a limited environment, such as an open field or a maze. Geometrically, one or two cues could define a single place in a circular arena, if the boundaries are visible or otherwise perceived and subjects can evaluate their distance from the cue (Collett, 1987; Collett, Cartwright, \& Smith, 1986). Many papers, however, report no place discrimination in the presence of a single local cue (Pico, Gerbrandt, Pondel, \& Ivy, 1985; Scharlock, 1954, 1955). The white cue card often used in place unit electrophysiological studies (Taube et al., 1990) provides more information than a unique punctate visual cue, because it has at least two discrete black and white contrasts. Such a cue would also allow place discrimination in an arena. In the dark, two distinct visual cues support accurate place discrimination (Fenton et al., 1994) in rats previously trained to find the platform at a certain distance from the wall. However, it might depend on whether the cues are similar or different from each other. Two cues of different shapes placed on different heights on the horizon provide more spatial information than two identical cues at the same distance and height. If two distant cues are completely identical and perceptible simultaneously from the goal location, one might assume that they represent a snapshot image that would help discriminate a particular location in an experimental field, because their retinal image viewed from the goal is unique. However, the question remains as to whether two identical visual cues might serve as relational cues (Eichenbaum, Stewart, \& Morris, 1990)-that is, help identify the position of other places in the arena relative to that of the target.

We designed a specific experimental setup to analyze the ability of rats to use visual cues in a spatial memory representation. The first question was whether rats could use three identical light cues to learn the position of an escape hole in the dark. The second question was whether these cues need to be simultaneously available or whether rats might combine information perceived in different regions of the arena at different times. The third question (see Experiment 2) was whether rats would be able to discriminate a specific goal location in total darkness after prolonged training and whether that prolonged training would prevent any further improvement in spatial learning when visual cues from the environment became available.

\section{EXPERIMENT 1}

Rats learned to escape from a location in the central region of a homing table (Schenk, 1989) with the aid of controlled light cues. A triangular configuration of three cues identical in shape, distance from the table, and vertical position was used. In one condition, all three cues were permanently available from any part of the open field. In a second condition, two cues were on when the rat was in the periphery of the field. They were switched off when the rat entered the central region of the table, from which only the third light cue could be seen. Two other control conditions were used. In one, only the pair 
of cues was illuminated when the rat was in the periphery. In the other, only the single cue was available when the rat was in the center. These conditions are very similar to a natural situation, such as in a woodland area, in which not all visible cues are perceived from all parts of the environment. For further comparisons, spatial discrimination was also assessed in total darkness (no cue) or in normal illumination conditions (all distant visual room cues accessible).

In this experimental design, it is possible to compare spatial discrimination with three simultaneously available cues and discrimination with the same number of cues conditionally accessible from different regions of the table. It was expected that spatial discrimination of the training position was equally attainable with the set of three simultaneously or separately available cues, but not with any of the limited subsets (two or one cue only). This would indicate that spatial discrimination does not require a specific local view of all three cues to be seen from the training position, but that rats could attain accurate localization by combining information obtained at different times in different parts of the table. Other comparisons were aimed at assessing whether the discrimination shown with our controlled cues in darkness was different from that supported by the distant room cues.

\section{Method}

Subjects. Forty-four adult Long-Evans hooded rats (32 females and 12 males) were used in compliance with Swiss guidelines for the care and use of laboratory animals. They were housed in groups of 4 in large glass containers $(40 \times 40 \times 50 \mathrm{~cm})$, with a wooden nest box and various wooden pieces encouraging climbing and movements through holes. This can be considered as a semi-enriched rearing condition. Daily food rations were delivered immediately following testing and were adjusted to prevent any weight increase throughout the duration of the experiment (i.e., between 4 and 9 days). The animals were kept in a natural light:dark cycle and were tested in the afternoon.

Apparatus. A large circular board (diam., $170 \mathrm{~cm}$ ) with no wall was utilized (Schenk, 1989). The platform was provided with 15 possible escape holes grouped in five triplets, as is shown in Figure 1. Each hole was covered with a disk of light plastic, which had the same color as that of the platform and could easily be removed by the rats. The detachable fittings of the holes are interchangeable and washable. Only one hole allowed passage to the subject's home cage placed under the table. The fitting for the goal was connected to a flexible tube and hidden by a light plastic disk. Dead end holes were blocked with a thick piece of foam rubber hidden under the plastic disk. The empty home cage was placed under the table and connected to the flexible tube, which was curved in order to minimize the slope. The extremity of the tube (length, $50 \mathrm{~cm}$ ) was simply deposited in the open cage. The home cage and the connected tube could not be seen from the surface of the open field.

The table was lit by two infrared projectors (Infralite IR-RS 4060: narrowband light emission; wavelength, $880 \mathrm{~nm}$ ) placed in a false ceiling. An infrared video camera placed directly above the center of the table allowed recording of each test session. An observer was able to view the rat's movements through an infrared viewer (Find-R-Scope 85100, Cotraco Establishment).

Behavioral procedure. All the rats were pretrained, on a small platform in another room, to enter a covered hole connected to a tunnel until they removed the cover and spontaneously entered the hole. Daily sessions on the homing table consisted of five training trials. Pseudorandom sequences of five different starting positions on the periphery of the table were used in each training session. Individual rats remained on the table until they found the escape hole, designated at a fixed spatial location throughout training, or until $120 \mathrm{sec}$ had elapsed. Rats not able to locate the escape hole in the allotted time were gently guided, and, if necessary, the hole was uncovered. Spontaneous exploratory episodes after the rat had located the escape hole were also limited to the allotted time in the same manner. During the initial trials, the holes were partially masked by the covers, which the rats rapidly learned to push aside for entry into the connected hole. By the end of the second session, the holes were completely covered.

A drop of condensed milk was available in the home cage to maintain a stable escape motivation. After the rat had reached the cage and found the condensed milk, it was placed in a closed bucket for the 2-min intertrial interval. The position of the bucket was varied from trial to trial so that it did not represent a fixed spatial reference position. The table was rotated between trials in a nonsystematic fashion (random), and the escape hole and covering were replaced by clean ones. The intertrial rotation did not affect the configuration between the cues and the escape location but made olfactory traces inconsistent.

Probe trials were conducted to assess the spatial bias toward the training position in the different conditions. In these trials, the hole on the training position was not connected to the home cage and, therefore, was not different from the other holes. After $72 \mathrm{sec}$, the hole on the training position was connected, and the rat was allowed access to its home cage. An additional reinforced trial was given immediately following the probe trial, to compensate for possible extinction effects.

All the trials were video recorded and analyzed later. The latency to uncover the escape hole (escape latency) was recorded, and an error was scored when the rat uncovered an incorrect hole. The trials of Sessions 4 and 6 and all the probe trials were analyzed by an XY videotracker. The distance covered to reach the correct hole (escape distance) was calculated. The time elapsed in each hole sector (diam., $20 \mathrm{~cm}$ ) during the probe trials was calculated from recordings of the 72-sec period during which the hole remained unconnected.

Experimental conditions. Six different conditions were created, depending on whether the rats were trained with the usual indirect lights (the light condition), in complete darkness (the no-cue condition), or in the dark with controlled light cues. Each cue was made of a row of three light-emitting diodes. The cues were placed $50 \mathrm{~cm}$ from the table limit and at the platform level, as is indicated in Figure 1. Four different cue-controlled conditions were used, depending on the number of available cues. For one condition, three identical cues were permanently on (the 3-cues condition). For the three remaining conditions, the cues were on or off, depending on the rat's position on the table, which was determined by using a videotracker (Kukam, Lausanne) coupled to a computer. In one condition (the 2+I-cues condition), a single cue (see Figure $1 \mathrm{~b}$ ) was on whenever the rat was in the central part of the table, whereas two other cues were on when the rat was in the periphery of the table. No cue was on when the rat (more precisely, the center of gravity of its dark contrasted head and shoulders as detected by the tracker) was in a 5 -cm-wide annulus between the center and the peripheral zones. In this way, the three cues were never simultaneously on. In one condition, a single cue was on when the rat was in the center of the table ( 1 -cue-c condition), and none when the rat was in the periphery. In another condition, two cues were to be seen from the periphery and none from the center (the 2-cues- $p$ condition). When the three cues were on, the light intensity on the apparatus was less than 0.001 lux.

Experimental design. Six different groups of rats were trained according to the conditions described above. The light, no-cue, and 3 -cues groups were considered to be control groups and were, therefore, composed of a larger number of subjects of both sex, in order to be representative of the rats in general. The experimental l-cue-c, 

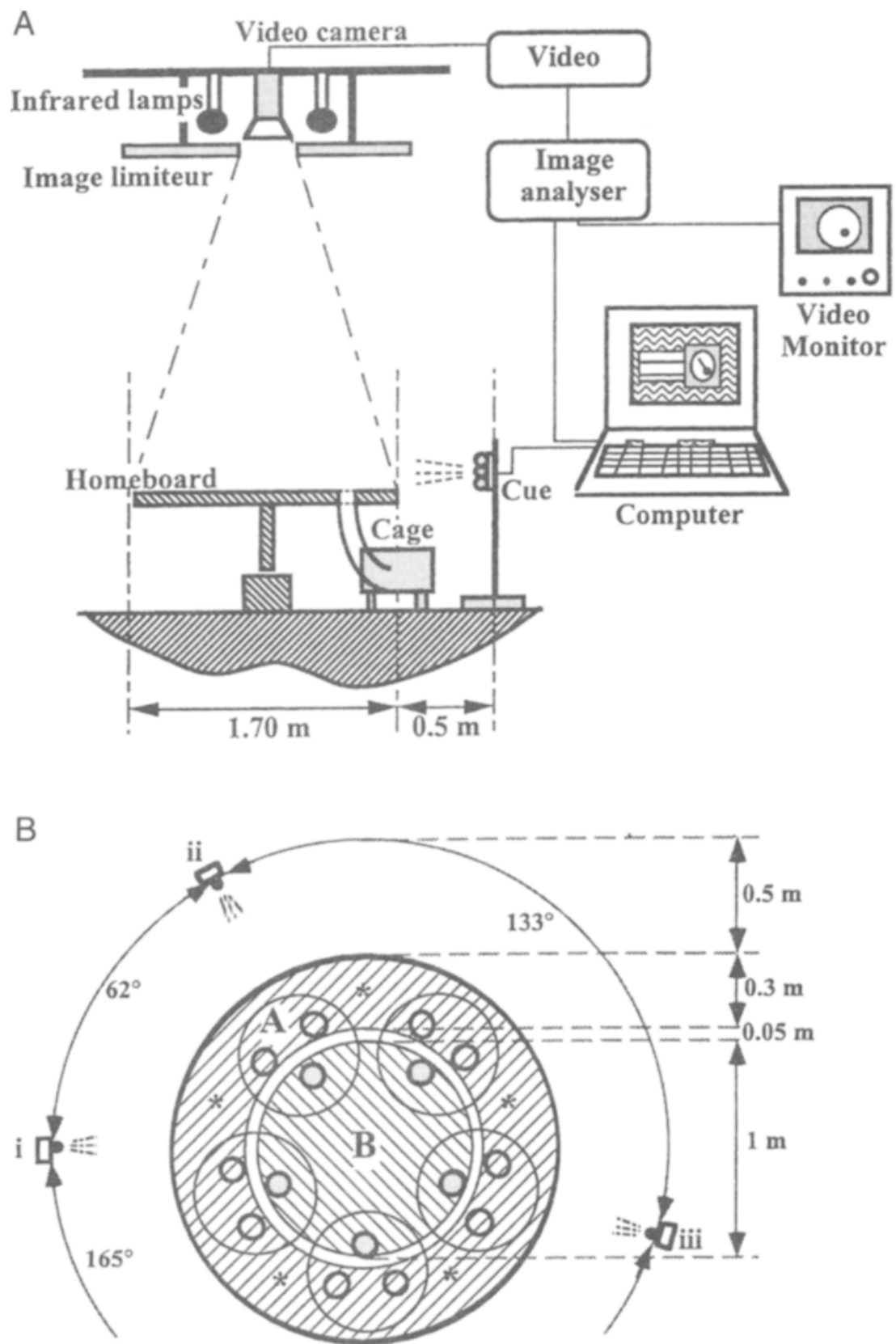
DDA A. periphery of the board (cues $i$ and ii were the associated one)
IIV B. central part of the board (the cue iii is the associated one)

\section{* Entrances \\ O 5 escape holes which were used}

Figure 1. Panel A: plan of the homing table with the position of the light cues. Panel B: overview of the homing hole board and of the cue's disposition.

2-cues-p, and 2+1-cues groups were each composed of 6 females. No males were included in these groups, because no gender difference was found in the control groups. All the groups were trained during 4 days, with 5 training trials each day ( 20 training trials). They were given two standard probe trials with unchanged cue condition at the end of the 2 nd and 4 th training sessions (see Table 1).
Three groups were further trained. The 3-cues group was given five additional training sessions (for a total of 45 trials), including 2 probe trials. One probe trial was conducted at the end of Session 6 , following a $+90^{\circ}$ rotation of the cues. A second probe trial was conducted at the end of Session 9, with a single cue that provided more light. The 2 -cues-p and $2+1$-cues groups were given 
Table 1

Experimental Design

\begin{tabular}{|c|c|c|c|c|c|c|c|c|c|}
\hline \multirow[b]{2}{*}{ Condition } & \multicolumn{9}{|c|}{ Sessions } \\
\hline & Day 1 & Day 2 & Day 3 & Day 4 & Day 5 & Day 6 & Day 7 & Day 8 & Day 9 \\
\hline Light & 5 trials & 5 trials & 5 trials & 5 trials & & & & & \\
\hline$(n=8 ; 4 \mathrm{~F} \& 4 \mathrm{M})$ & & T1 std & & $\mathrm{T} 2$ std & & & & & \\
\hline No-cue & 5 trials & 5 trials & 5 trials & 5 trials & & & & & \\
\hline$(n=10 ; 6 \mathrm{~F} \& 4 \mathrm{M})$ & & T1 std & & T2 std & & & & & \\
\hline 1 -cue-c & 5 trials & 5 trials & 5 trials & 5 trials & & & & & \\
\hline$(n=6 ; 6 \mathrm{~F})$ & & $\mathrm{T} 1$ std & & $\mathrm{T} 2 \mathrm{std}$ & & & & & \\
\hline 2-cues-p & 5 trials & 5 trials & 5 trials & 5 trials & 5 trials & 5 trials & 5 trials & & \\
\hline$(n=6 ; 6 \mathrm{~F})$ & & T1 std & & $\mathrm{T} 2 \mathrm{std}$ & & T3 std & T4 std & & \\
\hline $2+1-$ cues & 5 trials & 5 trials & 5 trials & 5 trials & 5 trials & 5 trials & 5 trials & & \\
\hline$(n=6 ; 6 \mathrm{~F})$ & & T1 std & & T2 std & & $\mathrm{T} 3$ rotation & T4 2-cues-p & & \\
\hline $\begin{array}{l}3 \text {-cues } \\
(n=8 ; 4 \mathrm{~F} \& 4 \mathrm{M})\end{array}$ & 5 trials & $\begin{array}{l}5 \text { trials } \\
\text { T1 std }\end{array}$ & 5 trials & $\begin{array}{l}5 \text { trials } \\
\text { T2 std }\end{array}$ & 5 trials & $\begin{array}{l}5 \text { trials } \\
\text { T3 rotation }\end{array}$ & 5 trials & 5 trials & $\begin{array}{l}5 \text { trials } \\
\text { T4 single-cue }\end{array}$ \\
\hline
\end{tabular}

Note-T std, standard probe trial; $T$ rotation, probe trial with the light cues displaced; $T$ 2-cues p, probe trial with no central cue; $T$ single-cue, probe trial with 2 cues off and one cue providing more light; $F$, female; $M$, male.

three additional training sessions (for a total of 35 trials). The 2cues-p group was given 2 standard probe trials, at the end of Sessions 6 and 7 . The $2+1$-cues group was given 1 probe trial following a $+90^{\circ}$ rotation of the cues at the end of Session 6 . For this latter group, training during Session 7 was conducted without the central cue being turned on. A probe trial was given at the end of this session with this restricted cue condition. The detailed design is indicated in Table 1.

Statistics. Exact $p$ values will not be indicated in the results section. The .05 level was used as the criterion for statistical significance.

\section{Results}

Training trials. The escape latency decreased throughout training, indicating that all the groups learned to escape through the connected hole. No differences were found between females and males for the three control groups (light, 3-cues, and no-cue groups). Figure $2 \mathrm{~A}$ reveals that only the group trained in the light had shorter escape latencies than the groups trained in the dark with no light cues. A two-way repeated measures analysis of variance (ANOVA) was conducted with the six groups over the first four training sessions. There was a significant decrease in latency over training $[F(3,114)=53.9]$, a significant group effect $[F(5,114)=3.31]$, and a significant interaction between groups and training sessions $[F(15,114)=1.78]$.

The group trained in the light had significantly shorter escape paths during Session 4 (Figure 2B). A one-way ANOVA conducted on the mean escape distance in Session 4 indicated that the light group had shorter escape paths than the other groups (comparisons between the light group and all the other groups were significant). ANOVAs conducted with only the five groups trained in the dark (the no-cue, 3-cues, 2-cues-p, 1-cue-c, and 2+1cues groups) revealed no significant effect of the presence of the light cues on either the escape latency or the path length during Session 4. The number of errors (uncovering incorrect holes) was also the lowest in the light group, but the ANOVA conducted with the six groups was not significant.
Training was extended in undisturbed conditions for the 3-cues, 2-cues-p, and 2+1-cues groups. Figure 2A shows a steady decrease in escape latencies. However, the latencies of the 3-cues and 2-cues-p groups recorded in Session 6 were still higher than those of the light group in Session 4 (Fisher tests significant). This was no longer the case for the $2+1$-cues group.

Standard probe trials. During the standard probe trials, the rats were left on the table for $72 \mathrm{sec}$ while the escape hole was not connected. Two such probe trials were conducted, at the end of Sessions 2 and 4. Statistical analyses were based on mean results ( $\mathrm{T} 1$ and $\mathrm{T} 2$ were combined for each rat, since there was no difference between the discrimination in both trials). Figure 3 shows that the rats in the light condition or in the conditions with three cues (3-cues and 2+1-cues conditions) spent significantly more time in the target sector than in the nontarget sectors. A two-way ANOVA, with time spent in each sector (repeated factor) and conditions group as factors, confirmed a significant effect of group $[F(5,38)=3.6]$ and of sector $[F(4,20)=11.22]$ and a significant interaction between the factors $[F(20,152)=2.76]$. A one-way ANOVA of the relative time spent in the training sector by each group confirmed the significant effect of training condition $[F(5,38)=4.67]$. Those groups with three cues or in the light condition spent significantly more time in the training sector (the light, 3-cues, and 2+1-cues groups). The three groups with fewer than three cues did not significantly discriminate the training location.

The times spent in the central region and in the periphery of the table were compared during the probe trials. The two groups for which the cues were accessible from one region of the table only (the 1-cue-c and 2-cue-p groups) spent about $50 \%$ of their time in the center and $50 \%$ in the periphery of the table. The amount of time spent in the periphery or in the center was not significantly affected by access to the light cue.

Probe trial following rotation of the light cues. During these probe trials for the 2+1-cues and 3-cues groups, 
A

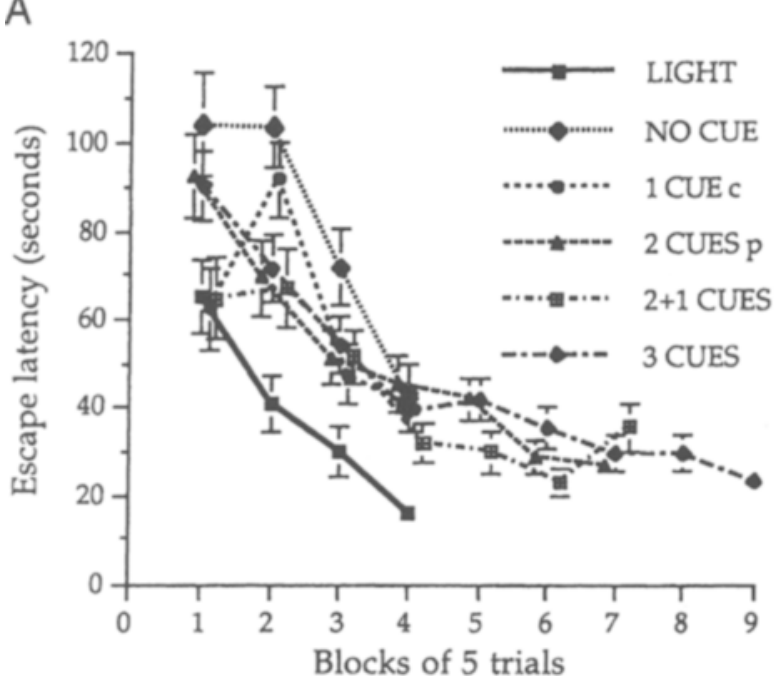

B

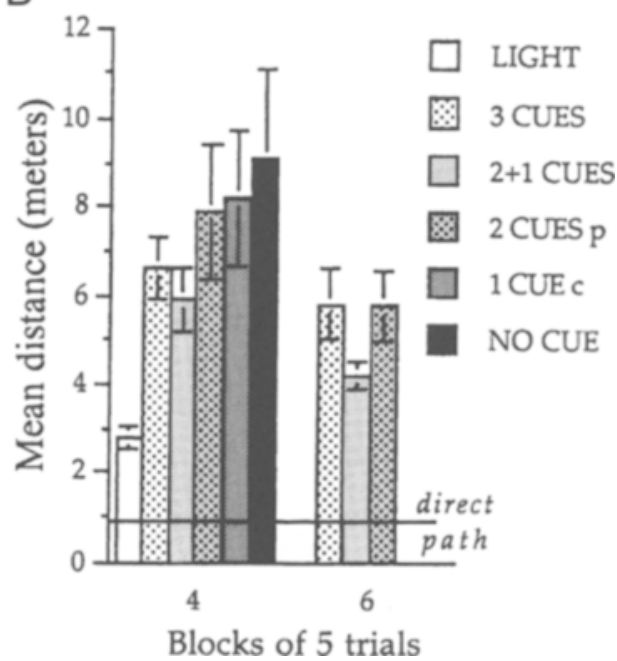

Figure 2. Panel A: escape latency through training sessions. Panel B: mean path length (escape distance) during Sessions 4 and 6.

the light cues were rotated by $90^{\circ}$ and the position of the table was adjusted so as to bring a hole triplet in the new position defined by the cues. Figure 4 shows that the representative rats in the groups with three cues (the 3-cues and 2+1-cues groups) spent more time near the cuedefined training position. The behavior of rats in the $2-$ cues- $p$ condition was more erratic, although no cue rotation had occurred. Discrimination of the training sector was only significant in the groups with three cues, supported by a significant interaction between the training condition and the sector effect [two-way ANOVA, with sector as a repeated measure, $F(8,68)=2.61]$. In fact, oneway repeated measures ANOVAs revealed that the $2+1-$ cues group spent significantly more time in the training sector $[F(4,29)=8.76]$, as did the 3 -cues group $[F(4,39)=$ $4.84]$, whereas the 2 -cues-p group did not spend more time in the training sector.

Probe trials with a restricted number of visual cues. The groups trained in the 2-cues-p, 2+1-cues, and 3-cues conditions were given extended training in the same condition. The last trial was a probe trial, with an unchanged condition for the rats in the 2-cues-p group. The number of available cues was reduced for the other groups (only two cues visible from the periphery for the second group, only one brighter cue for the group previously trained with three permanently available cues). Figure 5 shows that the discrimination of the training sector had not improved with prolonged training in the first (2-cues-p group). Moreover, the reduction of the number of available cues appears to prevent the discrimination of the training sector in both groups (no significant discrimination of the training sector in either group).

\section{Discussion}

Task acquisition was observed in all the training conditions. The rats learned that one of the holes allowed es- cape into the home cage, as is indicated by a progressive and significant decrease of escape latencies. However, the group trained with indirect room illumination showed significantly lower escape latencies and shorter escape paths than did the groups trained in the dark with no cue or with any of the limited-cue conditions. Moreover, all of the other groups had similar escape latencies and path lengths. Thus, there was a major difference in acquisition performance between light and darkness conditions when the training hole was connected with the home cage. This suggests that limiting the amount and quantity of relevant spatial information diminishes the accuracy of the spatial navigation performance.

Performance during the probe trials revealed differences among the groups given limited-cue training. The discrimination of the training location during Probe Trials 1 and 2 was comparable for the two groups trained in the dark with three cues and for the group in the light condition, and all expressed significant search biases for the target sector. In contrast, the 1-cue-c, 2-cues-p, and no-cue groups did not express a sector bias on probe trials. For the 2+1-cues and 3-cues groups, place discrimination was based on the controlled cues, as demonstrated by the third probe trial following cue rotation. The similar performances of these two groups show that the dissociated access to visual cues did not reduce spatial discrimination performance. The rats trained with fewer than three cues showed no evidence of discrimination of the goal location, despite prolonged training. Furthermore, reducing the number of accessible cues for groups previously trained with three cues disrupted spatial discrimination, since the cue deletion manipulation eliminated target bias on the probe test.

The reduction of performance, between the group in the light condition and the two groups with three light cues, has such an amplitude that it may not be possible 


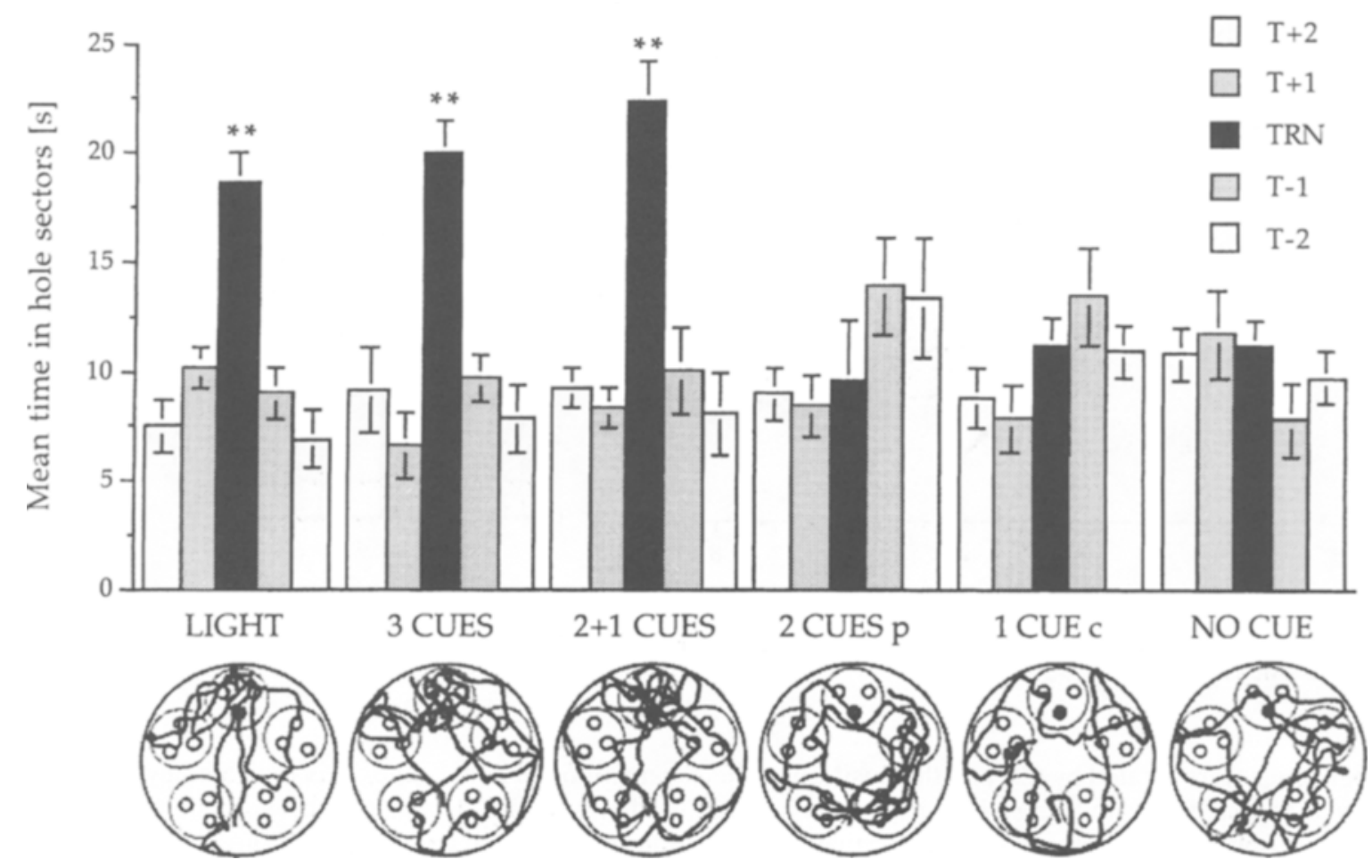

Figure 3. Top: mean(s) of the relative time spent $( \pm S E M)$ in each hole sector during Probe Trials 1 and 2 with no hole connected (TRN, training sector; $T+1$ and $T-1$, adjacent sectors; $T+2$ and $T-2$, opposite sectors). The discrimination of the training sector in the different groups is based on separate one-way analyses of variance of the absolute time in each hole sector $\left({ }^{* *} p<.01\right)$. Bottom: representative path by a rat in each group during the first probe trials (for each record, the training position is at 12 o'clock).

to distinguish, in darkness, spatial navigation from a systematic strategy, in terms of errors or even in terms of time spent to reach the escape hole. This could reasonably explain why we are not able to distinguish the groups with three cues from the groups with fewer than three cues.

From this first experiment, we conclude that rats are able to use three identical light cues to learn the position of an escape hole in the dark. The three light cues need not be simultaneously available. Rats seem to acquire place discrimination by combining two kinds of information, each insufficient to support spatial discrimination by itself. The spatial bias for the target sector expressed by the $2+1$-cues group appears to reflect conjoined use of two separate sets of spatial information successively integrated over the training experience into a unique spatial representation.

It is difficult to evaluate precisely the good performance of the groups with the controlled light cues after prolonged training, because the reference group trained with no cue received only limited training. So it seemed important to examine in a second experiment how the animals would behave in total darkness after prolonged training.

\section{EXPERIMENT 2}

In Experiment 1, the rats were trained in total darkness for only 4 days, and it might be that a prolonged training period would lead to an improved discrimination based on hypothetical uncontrolled cues. Thus, the aim of the second experiment was to examine whether prolonged training in total darkness would allow acquisition of discrimination of the training location.

The second experiment is important as a reference for the groups with the controlled light cues of Experiment 1 , given the prolonged training. To the extent that the rats in this experiment fail to show evidence of spatial discrimination at the end of prolonged training in total darkness, we can assume that the high performance after prolonged training of the groups with three cues (Experiment 1) during the probe trials can be attributed to the controlled cues and not to the prolonged training.

\section{Method}

Subjects. Nine adult Long-Evans hooded rats $(5$ females and 4 males) were used. Daily food rations were delivered immediately following testing and were adjusted to prevent any weight increase throughout the duration of the experiment ( 11 days). The animals were kept in a natural light:dark cycle and were tested in the afternoon.

Apparatus. The apparatus, the behavioral procedure, and the experimental conditions were the same as those in Experiment 1.

Experimental design. A unique group of 9 rats was trained successively in two conditions. The rats were all trained first for 7 days (five trials per day) in total darkness (the no-cue condition) and then for 4 days under normal light condition (the light condition). They were given three standard probe trials in total darkness at the end of 


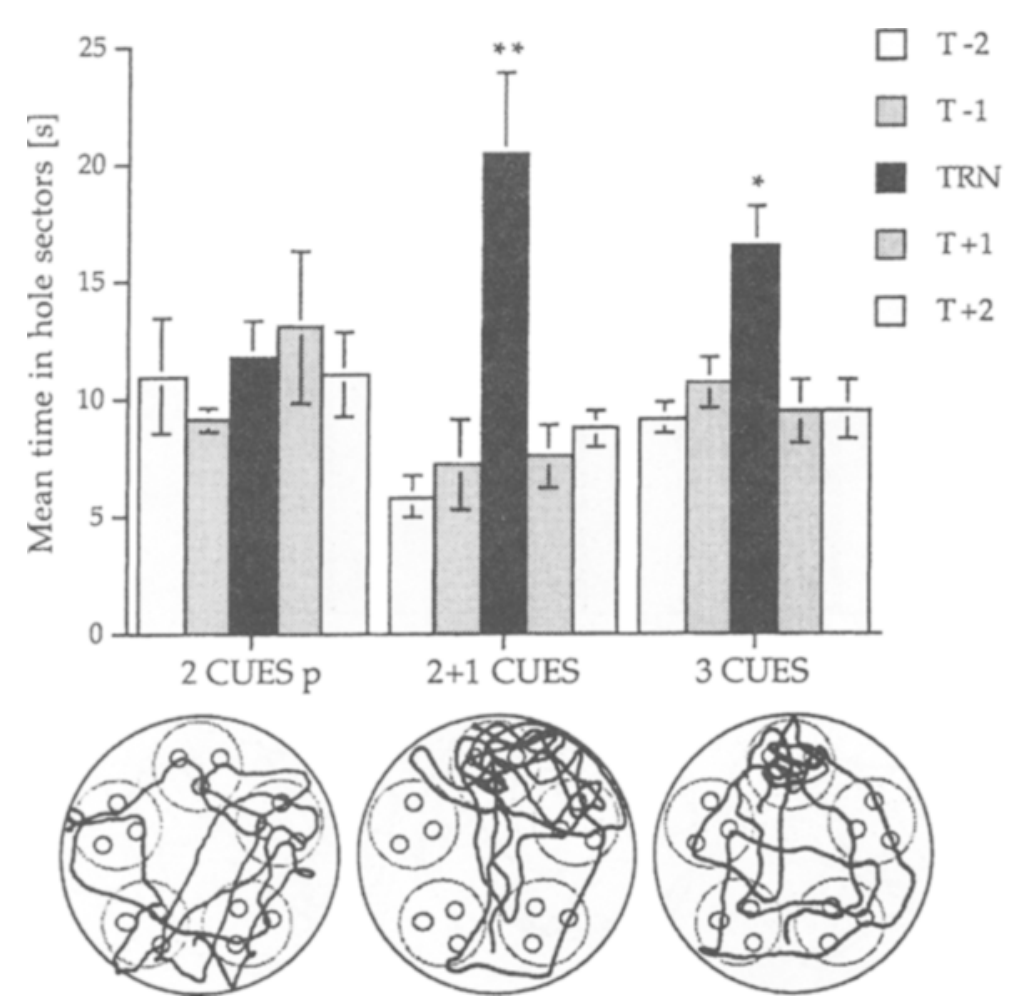

Figure 4. Top: means of the relative time spent $( \pm S E M)$ in each hole sector during the probe trials with no hole connected following a $90^{\circ}$ rotation of the cue (TRN, training sector; $T+1$ and $T-1$, adjacent sectors; $T+2$ and $T-2$, opposite sectors). The discrimination of the training sector in the different groups is based on separate one-way analyses of variance of the absolute time in each hole sector $\left({ }^{*} p<\right.$ $.05 ;{ }^{*} p<.01$ ). Bottom: representative path (for each record, the training position is at 12 'clock) by a rat in each group during the probe trial following displacement of the cue $\left(90^{\circ}\right.$ change of position).

the 2 nd, 4 th, and 7 th training sessions and two standard probe trials under light condition at the end of the 9th and 11 th training sessions. One probe trial was conducted in total darkness at the end of Session 6, following a $+90^{\circ}$ rotation of the apparatus. Escape distances were analyzed for Sessions $4,6,7,8$, and 11 .

Statistics. The criterion was the same as that in Experiment 1.

\section{Results}

Training trials. All the rats learned to escape through the connected hole in total darkness, indicated by the significant decrease in escape latency during the seven first sessions [Figure $6 \mathrm{~A} ; F(6,63)=12.95]$. There was no significant difference between females and males, and there was no significant reduction in latency from Session 4 to Session 7. During further training with illumination of the room cues (Sessions 8-11), there was a significant decrease in escape latency $[F(3,36)=13.58]$ but still no significant difference between females and males. The change of the light condition, in Session 8, did not induce any change of the latency.

A two-way ANOVA shows that there was no significant change in the escape distances between Sessions 4 and 7 (Figure 6B). But at Session 11, the rats had significant shorter escape paths, as compared with Session $8[t(8)=$
6.66]. No difference between females and males was found.

Probe trials. The rats were not able to discriminate the correct location in total darkness. A two-way ANOVA for the probe trials combined across Sessions 2 and 4 confirmed that the rats did not spend more time searching in the training area than in the other sectors (see Figure 7). During the probe trial following the rotation of the apparatus and the probe trial of Session 7, the rats were also not able to discriminate any location. There was also no difference between females and males.

When the room cues were illuminated (the light condition), the rats had no difficulties in discriminating the training area. They spent highly significantly more time in the training location during the combined probe trials of Sessions 8 and $11[F(4,45)=50.79]$. There was no differences between females and males $[F(1,45)=0.778]$.

\section{Discussion}

These results indicate clearly that, in our conditions, the rats did not show any discrimination of the training position on probe trials following prolonged training in total darkness, despite the fact that no attempt was made 


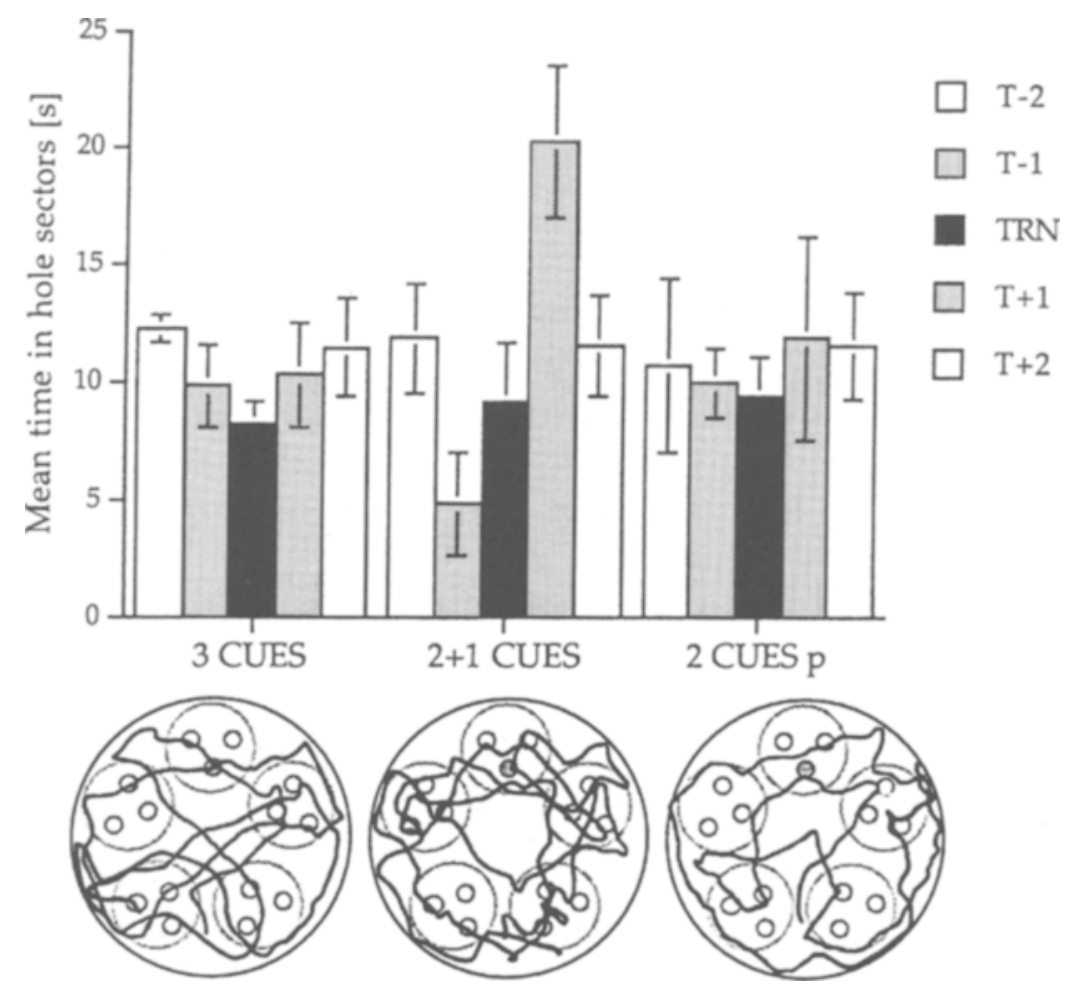

Figure 5. Top: means of the relative time spent $( \pm S E M)$ in each hole sector during the probe trials with no hole connected and with cue deletion (TRN, training sector; $T+1$ and $T-1$, adjacent sectors; $T+2$ and $T-2$, opposite sectors). The $T+1$ adjacent sector during Probe Trial 4 was the training sector during the previous training trial. The discrimination of the training sector in the different groups is based on separate one-way analyses of variance of the absolute time in each hole sector. Bottom: representative path by a rat in each group during the probe trials with no hole connected and with cue deletion (for each record, the training position is at 12 o'clock).

to control auditory or other unknown cues. The decrease in escape latency observed during the seven training sessions was accompanied by a decrease in escape distance. However, escape distances remained highly variable and were around $4.5 \mathrm{~m}$, whereas the length of straight escape path was about of $85 \mathrm{~cm}$. The poor spatial performance was confirmed by the systematic lack of discrimination during all the probe trials conducted in darkness.

Further training with indirect room illumination led to a progressive decrease in escape latency and distance, coupled with a significant discrimination of the training sector. The slow improvement suggests that the rats learned to identify and to reach the training location and that they were not simply motivated to reach the training hole because of an increased illumination level.

\section{GENERAL DISCUSSION}

Rats trained in total darkness showed no sign that they could discriminate the position of the training hole. Neither did the presence of one or two visual cues support place discrimination. It is concluded that any uncontrolled (auditory or otherwise perceptible) cues present in the test situation were insufficient for place discrimination to occur. Uncontrolled olfactory cues on the table were made irrelevant by intertrial rotations of the apparatus. The odor of the connecting tube might have facilitated identification of the escape position during the training trials in all experimental conditions. During the probe trials, however, there was no connection, and the hole at the escape position was similar in that respect to any other hole on the table.

Groups provided with three light cues, whether permanently on (3-cues condition) or contingent on the rat's position ( $2+1$-cues condition), expressed a significant spatial bias toward the training position defined by the light cues. Indeed, the spatial relevance of these light cues was confirmed in probe trials following cue rotation.

However, there was an unexpected dissociation between the escape latency during the training trials and the spatial bias during the probe trials. During the probe trials, the groups trained with three light cues showed the same clear-cut discrimination of the training area as that expected from training in the illuminated room. During the training trials, however, these groups had longer latencies and escape distances, as compared with the group 

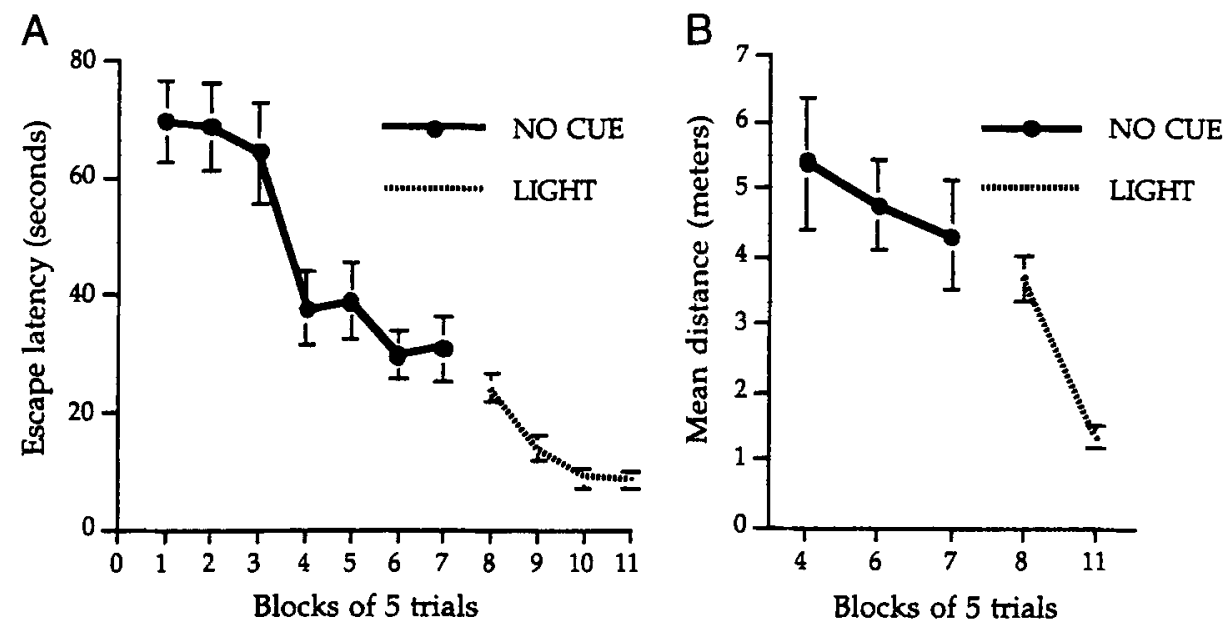

Figure 6. Panel A: escape latency through training sessions. Panel B: mean path length (escape distance) during Sessions 4, 6, 7, 8, and 11 .

trained in the illuminated room. In fact, all the groups trained in darkness had prolonged escape paths, irrespective of cue availability. Thus, cue-based discrimination of the training position was only significant in the probe trials. This discrepancy might be due to some differences in task demands during escape or probe trials.

To succeed in the escape trials, rats need rapid identification of their starting position, so they can head for the target hole. During the intertrial intervals, the rats were maintained in a closed bucket, the position of which was different during successive intertrial intervals. The fact that all the groups trained in darkness followed longer escape paths might be the result of an increased difficulty in identifying the starting position when only a limited set of visual cues was provided. Thus, after having been placed on the table, rats might have needed to walk a certain distance before they were able to identify their position, relative to the visual cues. This would be instantaneous in a cue-rich environment, such as that provided by room illumination. Consequently, task difficulty appeared greater at this stage, at which only few environmental cues were provided to recognize the starting position, so that no cue-related difference would appear among groups trained in darkness. The advantage for the group trained in the illuminated room would be to spend less time in identifying the starting position. Moreover, all the rats could eventually find the connected hole by chance or by following any systematic path from hole to hole. The uncovering of the correct hole (avoiding uncovering nonbaited holes) could be triggered by the odor of the connecting tube.

In contrast, during the probe trials, all the holes were similar, since the connection was not installed. No olfactory cue whatsoever could trigger the uncovering of the hole at the correct position. Thus, the three light cues played a major role for the discrimination of the training position during the normal probe trials and a critical one in the trials with rotation. Accordingly, a clear-cut spatial bias was only observed in the groups trained with at least three visual cues. Rats trained with less than three cues or no cue showed no discrimination of the training location. The accurate discrimination during the probe trials despite prolonged escape latencies indicates that our discrete cues do not allow for a short, nearly direct escape path from the training position during the training trials. These cues are, nevertheless, able to promote a clear identification of the escape position.

The longer latencies in darkness with three light cues confirm the previous finding by Liu, Turner, and Bures (1994), suggesting that, during locomotion, it might be necessary for the rats to see the light cues for a prolonged time, in order to allow computation of the target position. This increased delay should not interfere with the discrimination of the training position, as measured from the time spent in this area during the probe trial, but may explain why rats took longer escape paths in the dark.

These results confirm that a few discrete light cues allow spatial navigation in the dark, as was shown by Fenton et al. (1994). In our experiments, three identical point light cues were necessary to support accurate spatial discrimination. In contrast, Pico et al. (1985), as well as Fenton and his colleagues, found that two different cues were sufficient to support spatial navigation in a cross maze and in a circular pool, respectively. In our experimental design, removal of the single light cue in the $2+1$-cues condition impaired spatial discrimination in a significant manner. This suggests that, even after prolonged training in this condition, the rats were not able to obtain enough information from the two cues seen in the periphery, in the absence of the centrally available cue.

The discontinuous access to the light cues did not affect the rats' spatial performances. The discrimination of the training sector was equally accurate, whether the three cues were permanently on or whether their availability was conditioned on the rat's position. This indicates that the discrimination was not based on a simple snapshot 


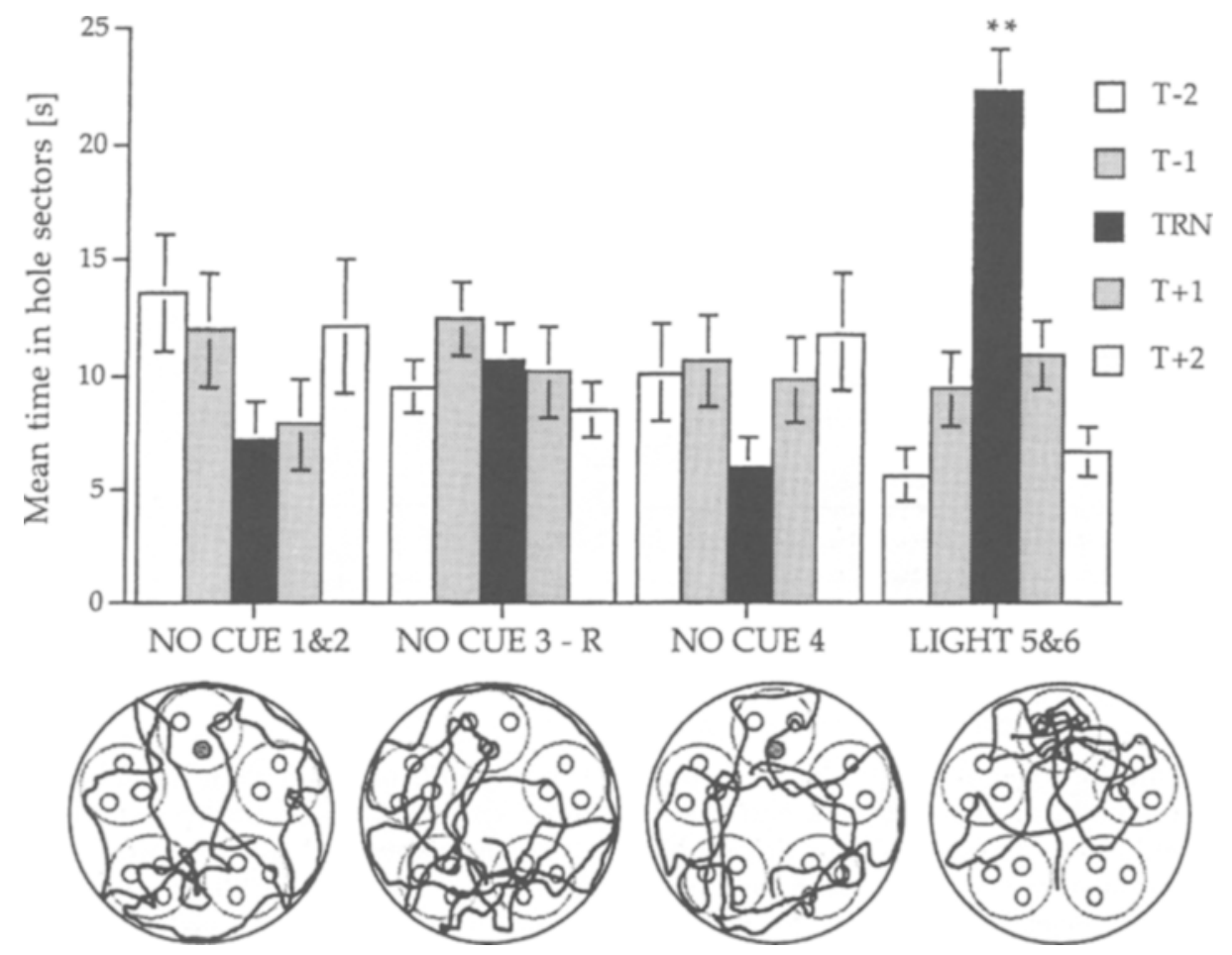

Figure 7. Top: means of the relative time spent $( \pm S E M)$ in each hole sector during the different probe trials (TRN, training sector; $T+1$ and $T-1$, adjacent sectors; $T+2$ and $T-2$, opposite sectors). The discrimination of the training sector for the different probe trials is based on separate oneway analyses of variance of the absolute time in each hole sector $(* * p<.01)$. Bottom: representative path by a rat for each first probe trial.

memory of the position of the cues, as seen from the training hole, but that rats could rely on memory for the position of the cues seen in other parts of the arena and, consequently, at different moments. This memory apparently bridges both a temporal gap and a spatial gap. In fact, even in the 3-cues condition, all the cues were probably not seen simultaneously, owing to their relative position (see Figure 1). Thus, it is not likely that they allowed for a simple adjustment between a perceived image and a memorized image, as is suggested in the simple snapshot memory model of Wilkie and Palfrey (1987). Moreover, when the cues were only accessible from some parts of the arena, as in the 2+1-cues condition, there was a spatial gap between perception of different cues, and rats had to refer their own position to that of the set of cues in an even more abstract way. Thus, animals perceiving two sets of cues at different places and at different moments appear to be able to integrate successively those two pieces of insufficient spatial information and to cumulate that information, in order to achieve a place discrimination task.

Our results are in line with different experiments indicating that rats can maintain accurate place discrimination when the light goes off (Quirk, Muller, \& Kubie, 1990; Schenk et al., 1995), when they are in a homogenous arena (Schenk et al., 1997), when all the cues forming a controlled environment have been removed (O'Keefe \& Speakman, 1987), or when ambiguity among identical visual cues can only be solved by path integration (Sharp et al., 1990).

From these results, it appears that rats are able to encode the spatial relationships between a limited number of visual landmarks despite their discontinuous availability. These isolated pieces of information are integrated into a spatial representation that may be used to discriminate a precise emplacement. To understand the rules underlying the integration of visual information into abstract representations and the abstractness of this type of representation, further experiments employing different combinations of cues from vision and other sensory modalities, such as audition and olfaction, should be conducted.

\section{REFERENCES}

Arolfo, M. P., Nerad, L., Schenk, F., \& Bures, J. (1994). Absence of snapshot memory of the target view interferes with place navigation learning by rats in the water maze. Behavioral Neuroscience, 108 , 308-316.

BiegLer, R., \& Morris, R. G. M. (1993). Landmark stability is a prerequisite for spatial but not for discrimination learning. Nature, 361, 631-633.

Biegler, R., \& Morris, R. G. M. (1996). Landmark stability: Studies exploring whether the perceived stability of the environment influences spatial representation. Journal of Experimental Biology, 199, 187-193.

Buresova, O., \& Bures, J. (1981). Role of olfactory cues in the radial maze performance of rats. Behavioural Brain Research, 3, 405-409.

Cartwright, B. A., \& Collett, T. S. (1982). How honey bees use landmarks to guide their return to a food source. Nature, 295, 560-564. 
Chapillon, P. (1999). Very brief exposure to visual distal cues is sufficient for young mice to navigate in the Morris water maze. Behavioural Processes, 46, 15-24.

ChENG, K. (1986). A purely geometric module in the rat's spatial representation. Cognition, 23, 149-178.

Collett, T. S. (1987). The use of visual landmarks by gerbils: Reaching a goal when landmarks are displaced. Journal of Comparative Physiology A, 160, 109-113.

Collett, T. S., Cartwright, B. A., \& Smith, B. A. (1986). Landmark learning and visuospatial memories in gerbils. Journal of Comparative Physiology $A, 158,835-851$.

Eichenbaum, H., Stewart, C., \& Morris, R. G. M. (1990). Hippocampal representation in place learning. Journal of Neuroscience, 10, 3531-3542.

Fenton, A. A., Arolfo, M. P., Nerad, L., \& Bures, J. (1994). Place navigation in the Morris water maze under minimum and redundant extra-maze cue conditions. Behavioral \& Neural Biology, 62, 178-189.

Goodridge, J. P., \& TAube, J. S. (1995). Preferential use of the landmark navigation system by head direction cells in rats. Behavioral Neuroscience, 109, 49-61.

Greene, C. M.. \& CoOK. R. G. (1997). Landmark geometry and identity controls spatial navigation in rats. Animal Learning \& Behavior, 25, 312-323.

Grobéty. M.-C., Morand. M.. \& SChenk. F. (in press). Cognitive mapping in rats and humans: The tent-maze, a place learning task in visually disconnected environments. In S. O'Nuallain (Ed.), Proceedings of mind III. Benjamins.

Hetherington, P. A., \& Shapiro, M. L. (1997). Hippocampal place fields are altered by the removal of single visual cues in a distancedependent manner. Behavioral Neuroscience, 111, 20-34.

Knierim, J. J.. Kudrimoti, H. S.. \& McNaughton. B. L. (1995). Place cells, head direction cells, and the learning of landmark stability. Journal of Neuroscience, 15, 1648-1659.

LiU, Z., TURNER. L. F.. \& BURES. J. (1994). Impairment of place navigation of rats in the Morris water maze by intermittent light is inversely related to the duration of the flash. Neuroscience Letters, 180, 59-62.

Markus, E. J.. Barnes. C. A.. Mi Naughton, B. L.. Gladden. V. L.. \& SkAGigs. W. E. (1994). Spatial information content and reliability of hippocampal CAI neurones: Effects of visual input. Hippocampus, 4, 410-42i.

MoNaughton. B. L.. KNierim. J. J.. \& Wilson. M. A. (1995). Vector encoding and the vestibular foundations of spatial cognition: Neurophysiological and computational mechanisms. In M. S. Gazzaniga (Eds.), The cognitive neurosciences (pp. 585-595). Cambrige, MA: MIT Press.

Morris, R. G. M. (1981). Spatial localisation does not require the presence of local cues. Learning \& Motivation, 12, 239-260.

MULLER. R. U.. \& KuBIE. J. L. (1987). The effects of changes in the environment on the spatial firing of hippocampal complex-spike cells. Journal of Netroscience, 7, 1951-1968.
O'KeEfe, J., \& Burgess, N. (1996). Geometric determinants of the place fields of hippocampal neurones. Nature, 381, 425-428.

O'Keefe, J., \& Speakman, A. (1987). Single unit activity in the rat hippocampus during a spatial memory task. Experimental Brain Research, 68, $1-27$.

Pico, R. M., Gerbrandt, L. K., Pondel, M., \& Ivy, G. (1985). During stepwise cue deletion, rat place behaviors correlate with place unit responses. Brain Research, 330, 369-372.

Quirk, G. J., Muller, R. U., \& KuBIE, J. L. (1990). The firing of hippocampal place cells in the dark depends on the rat's recent experience. Journal of Neuroscience, 10, 2008-2017.

SAVE, E. (1997). The contribution of visual and inertial mechanisms to navigation in total darkness. Animal Learning \& Behavior, 25, 324-334.

ScHARLOC K, D. P. (1954). The effects of a pre-extinction procedure on the extinction of place and response performance in a T maze. Jour nal of Experimental Psychology, 48, 31-36.

SCHARLOCK, D. P. (1955). The role of extra-maze cues in a place and response learning. Journal of Experimental Psychology, 50, 249-250.

SCHENK, F. (1989). A homing procedure for studying spatial memory in immature and adult rodents. Journal of Neuroscience Methods, 26 , 249-258.

SCHENK, F., Grobétr, M.-C., \& Gafner, M. (1997). Spatial learning by rats across visually disconnected environments. Quarterly Journal of Experimental Psychology, 50B, 54-78.

Schenk, F., Grobéty, M.-C., Lavenex, P., \& Lipp, H.-P. (1995). Dissociation between basic components of spatial memory in rats. In E. Alleva, A. Fasolo, H.-P. Lipp, L. Nadel, \& L. Ricceri (Eds.), Behavioral hrain research in naturalistic and semi-naturalistic settings. Possibilities and perspectives (pp. 277-300). Dordrecht: Kluwer.

SharP, P. E., KubIE, J. L., \& Muller, R. U. (1990). Firing properties of hippocampal neurons in a visually symmetrical environment: Contributions of multiple sensory cues and mnemonic processes. Journal of Neuroscience, 10, 3093-3105.

Sutherland, R. J., Chew, G. L., Baker, J. C., \& Linggard, R. C. (1987). Some limitations on the use of distal cues in place navigation by rats. Psychobiology, 15, 48-57.

Sutherland, R. J., \& DYCK. R. H. (1984). Place navigation by rats in a swimming pool. Canadian Journal of Psychology, 38, 322-347.

Taube, J. S., Mull.er, R. U., \& Ranck, J. B. (1990). Head-direction cells recorded from the postsubiculum in freely moving rats: II. Effects of environmental manipulations. Journal of Neuroscience, $\mathbf{1 0}$, 436-447.

WILKIE, D. M., \& PALFREY, R. (1987). A computer simulation model of rats' place navigation in the Morris water maze. Behavior Research Methods, Instruments, \& Computers, 19, 400-403.

ZOLADEK, L., \& RoBERTS, W. A. (1978). The sensory basis of spatial memory in the rat. Animal Learning \& Behavior, 6, 77-81.

(Manuscript received April 6, 1999; revision accepted for publication September 20, 1999.) 